\title{
Modelado numérico y análisis experimental para flujos en un medio poroso homogéneo a través de suelos
}

\section{Numerical modeling and experimental analysis for flows in a homogeneous porous medium through soils}

\author{
Mayerly Duran-Pinzón \\ Ingeniera Mecánica (c) \\ Universidad Francisco de Paula Santander \\ Ocaña, Colombia \\ mduranpi@ufpso.edu.co \\ Pedro Julián García-Guarín \\ M.Sc Ingeniería Mecánica \\ Servicio Nacional de Aprendizaje SENA \\ Ocaña, Colombia \\ pjgarciag@ufpso.edu.co
}

\author{
Jorge Páez-Arango \\ Ingeniero Mecánico (c) \\ Universidad Francisco de Paula Santander \\ Ocaña, Colombia \\ jepaeza@ufpso.edu.co
}

\begin{abstract}
Resumen- Las empresas petroleras estudian el movimiento de aguas subterráneas para futuras búsquedas de campos de perforación o excavaciones a cielo abierto, también ha sido utilizada para la purificación del agua por medio de filtros de arena. En esta investigación se tuvo como objetivo determinar el comportamiento del flujo de agua en un medio poroso (arena). Una de las leyes más usadas para comprender este fenómeno es la Ley de Darcy, la cual dice que el caudal que atraviesa el medio poroso es directamente proporcional a las diferencias de presiones e inversamente proporcional a la distancia que debe atravesar el fluido. Se evaluó: gradiente de presión versus (caudal, diámetro y velocidad granel). Los resultados del modelo numérico se comparan con los resultados obtenidos experimentalmente para un permeámetro construido para este propósito. EI modelo numérico planteado se utiliza para variar la geometría del permeámetro y determinar las mejores condiciones del funcionamiento.
\end{abstract}

Palabras Clave- Ley de Darcy, medio poroso, permeámetro, simulación computacional.

\begin{abstract}
The oil companies study the movement of groundwater for future searches of drilling fields or open-pit excavations, it has also been used for the purification of water by means of sand filters. The objective of this research was to determine the behavior of the water flow in a porous medium (sand). One of the laws most used to understand this phenomenon is Darcy's law, which says that the flow through the porous medium is directly proportional to the pressure differences and inversely proportional to the distance that the fluid must
\end{abstract}

pass through. It was evaluated: pressure gradient versus (flow rate, diameter and bulk velocity). The results of the numerical model are compared with the results obtained experimentally for a permeameter constructed for this purpose. The numerical model proposed is used to vary the geometry of the permeameter and determine the best conditions of operation.

Keywords- Darcy's law, porous medium, permeameter, computational simulation.

\section{INTRODUCCIÓN}

La Ley de Darcy, propuesta hace 161 años, establece que la relación entre la velocidad de descarga y el gradiente hidráulico del flujo del agua en arenas es una invariante del material llamada coeficiente de permeabilidad o conductividad hidráulica, por el ingeniero hidráulico francés de nombre Henry Darcy quien estudió filtros de arenas para purificar el agua, lo que lo llevó a hacer una investigación sobre el movimiento de fluidos en un medio poroso, con base en un sistema diseñado con el nombre de permeámetro [1].

El estudio del movimiento de fluidos en medios saturados porosos es un tema de interés en muchos campos, tales como la geofísica, la física de la roca y la ciencia de los materiales. Los conceptos fundamentales sobre las relaciones ten- 
sión-deformación y la dinámica de la deformación sólidos monofásicos porosos totalmente saturados por un fluido se establecieron en los trabajos de Biot [2].

Esta ley es una generalización sobre las condiciones del flujo de agua en arenas subterráneas, donde se muestra el comportamiento del fluido en un medio poroso homogéneo, teniendo en cuenta el campo de presión y el campo de velocidad que este va a presentar, por ejemplo el flujo a través de materiales más gruesos, como arena y grava, hay un balance en magnitud entre las fuerzas inerciales y viscosas. En esta investigación se llevara a cabo la medición de la conductividad hidráulica por medio de un dispositivo (el permeámetro) de forma experimental y utilizando como herramienta la modelación computacional con el software Open Foam, identificando que se cumplan las condiciones de esta ley y la verificación experimental de estas mismas [3].

El estudio para medios porosos se realiza mediante dos tipos de escala diferentes: la escala continua sin poros y la escala de Darcy. En la escala continua sin poros se utiliza la ecuación de Navier Stokes, en la que se describe el movimiento del fluido para medios continuos, donde los efectos de los poros son despreciables, la cual se basa en la derivada material de la velocidad del fluido y un gradiente de velocidad que indica la conservación del fluido e incompresibilidad. La Ley de Darcy del medio poroso se estudia para un medio continuo y homogéneo cuya dimensión característica es del orden de centímetros donde está acoplado al gradiente de velocidad [4].

La Ley de Darcy es una de las piezas fundamentales de la mecánica de los suelos. A partir de los trabajos iniciales de Darcy, monumentales para la época, muchos otros investigadores han analizado y puesto a prueba esta ley. A través de los trabajos posteriores se ha podido determinar que mantiene su validez para la mayoría de los tipos de flujo de fluidos en los suelos. Para filtraciones de líquidos a velocidades muy elevadas y la de gases a velocidades muy bajas, la Ley de Darcy deja de ser válida. En el caso de agua circulando en suelos, existen evidencias abrumadoras en el sentido de verificar la vigencia de la Ley de Darcy para suelos que van desde los limos hasta las arenas medias. Asimismo, es perfectamente aplicable en las arcillas, para flujos en régimen permanente. Para suelos de mayor permeabilidad que la arena media, deberá determinarse experimentalmente la relación real entre el gradiente y la velocidad para cada suelo y porosidad estudiados.

Quien desarrollo la ley de propagación de esfuerzos en ondas a través de un medio poroso elástico conteniendo un fluido viscoso comprimido, estableció casos en los que es válida la Ley de Darcy para un flujo Poiseuilli. Encontraron que el fluido puede ser descrito utilizando cuatro números adimensionales. Leclaire y Cohen Ilevaron a cabo un estudio experimental de la transmisión de ondas elásticas en muestras de agua saturada con polvo de vidrio en un rango de temperatura -30 a $0^{\circ} \mathrm{C}$. Dos ondas longitudinales y dos ondas transversales se detectaron para velocidades del orden de 2300 m/s y 4000 m/s, los resultados se utilizan para predecir valores presentes en los estudios de Biot [5], [6]. Romaña estudio los límites de la Ley de Darcy en la que identifica condiciones en las que se cumple y verifica lo experimental de las condiciones en las que no ocurre este flujo. Los resultados son aplicables a suelos cohesivos, materiales granulados con diámetro mayor a materiales de arena. La proporcionalidad entre el gradiente hidráulico se mantiene hasta condiciones de velocidad muy elevadas.

Los medios porosos también pueden ser analizados utilizando una matriz de esfuerzos sobre el medio sólido poroso, y el agua líquida, las dos fases se toman en consideración, la fase porosa y la fase líquida del fluido. La fase líquida por lo general se toma un líquido incompresible el cual soporta únicamente esfuerzos debidos a la gravedad y se toman los efectos debidos a la capilaridad como despreciables [7].

En adición, Juncosa et al. explican que un medio poroso parcialmente saturado está compuesto por especies que pueden estar presentes en diferentes fases. Una fase es una región física y termodinámicamente homogénea. Las especies son los constituyentes de las fases. La fase principal que sirve de armazón del medio poroso es la matriz sólida, que actúa como esqueleto firme alrededor del cual se encuentran las demás fases (líquido y gas). Tanto la fase líquida como la gaseosa tienen mayor facilidad de movimiento por la estructura molecular que presentan, que las fases sólidas, las cuales pueden moverse por deformación en respuesta a variaciones en el estado 
tensional. En el presente documento se estudia la fase porosa y el efecto de la fase líquida, por lo que en el permeámetro (dispositivo utilizado para medir la conductividad hidráulica) se eliminan los residuos de aire presentes, donde los poros pueden dirigir el agua y la conductibilidad está en su máxima expresión [8], [9].

Entre las diferentes fases hay relaciones de equilibrio, debido a las diferencias de presiones internas; por únicamente utilizar la fase líquida se desprecian efectos de flujos de especies entre fases. Por lo tanto, el agua que se mueve a través del medio poroso en estado líquido puede ser calculada como una respuesta al gradiente hidráulico utilizando la Ley de Darcy.

\section{MATERIALES Y MÉTODOS}

El estudio se realiza en dos etapas, se plantea un modelo numérico el cual muestra los perfiles de presión y velocidad del permeámetro, y se establece un análisis experimental para caracterizar el permeámetro.

\subsection{Supuestos del modelo numérico}

Se tomaron los siguientes supuestos, generalmente adoptados para modelos de flujo de fluidos en medios porosos:

- El medio poroso es saturado por el fluido, es decir, la saturación con respecto a un fluido se define cuando el fluido desplaza todo el aire que se encuentra en el medio poroso.

- El fluido es incompresible, ya que su densidad permanece aproximadamente constante a lo largo de todo el flujo. Por lo tanto, el volumen de todas las porciones del fluido permanece inalterado sobre el curso de su movimiento cuando el flujo o el fluido es incompresible. En esencia, las densidades de los líquidos son constantes y así el de ellos es típicamente incompresible.

- Una matriz solida permanece en reposo durante el proceso de flujo de fluido, la cual es estacionaria y no cambia en ninguno de sus puntos.

- La masa del fluido se conserva, sin embargo, no sufre deformación por esfuerzos cortantes en toda su trayectoria.

- La velocidad de las partículas del fluido cumple con la Ley de Darcy, esta consiste en estudiar las propiedades de un flujo de agua a través de un lecho de arena variando la longitud de la muestra y la presión del agua en los planos superficial e inferior.

El fluido no está sujeto a procesos de difusión, por lo tanto, no hay pérdidas de partículas en el medio poroso debido al movimiento del fluido.

\subsubsection{Ecuaciones}

Los medios continuos se modelan atenuando la derivada del tiempo y añadiendo un término.

La ecuación de Navier-Stokes, (1) se realiza con dos balances, utilizando uno de la conservación general de cantidad de movimiento lineal y utilizando un balance de masa con el que se obtiene la ecuación de continuidad. Para un flujo en estado estable e incompresible, se cancelan los términos mostrados, despreciando la gravedad (2).

$$
\begin{gathered}
\rho \frac{\partial \varkappa_{i}}{\partial t}+u_{j} \frac{\partial u_{i}}{\partial x_{j}}=\frac{\partial P}{\partial x_{i}}+\mu \frac{\partial^{2} u_{i j}}{\partial x_{j}^{2}}+S_{i}+\rho g \hat{k} \\
\vec{\nabla} \cdot(\rho \overrightarrow{\mathbf{V}})=-\frac{\partial \not \partial}{\partial t} \quad(2)
\end{gathered}
$$

Por lo que la ecuación se reduce a (3) y (4).

$$
\begin{gathered}
u_{j} \frac{\partial u_{i}}{\partial x_{j}}=-\frac{\partial P}{\partial x_{i}}+\mu \frac{\partial \tau_{i j}}{\partial x_{j}}+S_{i} \\
\vec{\nabla} \cdot \overrightarrow{\mathbf{V}}=0
\end{gathered}
$$

Término transitorio: derivada local, representa la variación de la velocidad con respecto al tiempo.

- Término advectivo: representa el transporte de partículas de fluidos debido a los cambios de velocidad en la dirección del fluido.

- Cambio de presión: fuerza de presión impulsora del fluido.

- Matriz de esfuerzos cortantes del fluido.

- Pérdidas viscosas y pérdidas debidas a esfuerzos inerciales.

La velocidad en el medio poroso se calcula utilizando la Ley de Darcy (5) que es ampliamente usada para flujos laminares, la conductividad hidráulica se determinó experimentalmente diseñando un permeámetro construido para este propósito [3].

$$
\underline{U} \equiv \varepsilon \underline{v}=-\frac{1}{\mu} \underline{\underline{k .}}(\nabla p-\rho \underline{\underline{g}})
$$




\subsubsection{Programa de simulación OpenFOAM}

Es una herramienta de simulación numérica basada en esquemas de volúmenes finitos (básicamente, se trata de una biblioteca de utilidades para implementar un esquema de volúmenes finitos) especialmente orientada para la simulación numérica en mecánica de fluidos, donde el código del software es libre (con acceso, por tanto, al código fuente) programado en $\mathrm{C}++\mathrm{y}$ producido por Open CFD Ltda.

Este programa es capaz de resolver tanto flujos sencillos como complejos (incluyendo flujos turbulentos, fenómenos de transferencia de calor y reacciones químicas), así como problemas en otros campos (como problemas electromagnéticos). Una de las principales ventajas, al margen de su flexibilidad, es su capacidad para ser ejecutado en paralelo. La implementación del cálculo distribuido (basada en el uso de MPI y directamente explotable por parte de procesadores multinúcleo, clúster o redes de ordenadores) no añade ninguna dificultad al usuario, por lo que la capacidad de cálculo del código está únicamente limitada por el hardware disponible.

En la estructura del programa está formado por librerías, las principales son tutoriales y solvers. En tutoriales existen aplicaciones sobre casos básicos, de combustión, flujos comprensibles, métodos discretos, DNS (Direct Numerical Simulations Of Turbulence), electromagnetismo, financiero, transferencia de calor, flujo incomprensible, colisiones de partículas, multifase y análisis de esfuerzo, dependiendo la aplicación se escoge un solucionador de la librería solvers para cada uno de los casos presentados anteriormente [10].

La forma de resolver los diferentes casos se lleva a cabo durante tres etapas:

$\checkmark$ Pre-procesamiento: donde se enmalla el caso.

$\checkmark$ Solucionador: se escoge un solvers de acuerdo con la aplicación presentada en el caso.

$\checkmark$ Post-procesamiento: interfaz gráfica.

\section{Métodos de solución}

- Método de Gauss-Seidel.

- Método simple: algoritmo simple.

\section{Método Gauss-Seidel}

Los métodos de Gauss y de Gauss-Seidel son procedimientos iterativos para resolver simultáneamente ecuaciones no lineales. Tanto Gauss como Gauss-Seidel implican la formulación mostradas en (6) y (7)

$$
\begin{gathered}
X=F(x) \quad \text { (6) La fórmula iterativa } \\
X(n+1)=F((x(n))) \quad(7)
\end{gathered}
$$

En Gauss se calculan los nuevos valores de $x(n+1)$ a partir de los $x(n)$ obtenidos en la iteración anterior.

En Gauss-Seidel, los valores obtenidos son utilizados inmediatamente después de haber sido calculados, aunque no haya terminado la iteración en curso (mayor rapidez, suele llamarse Gauss-Seidel con actualización de variables) [10].

\section{Método simple}

El algoritmo simple tiene más de cuatro décadas de existencia, es numéricamente eficiente y estable, trata de fluidos incompresibles, la aplicación de este algoritmo ha sido utilizada para modelar todo tipo de flujos y geometrías. La filosofía del método es resolver las ecuaciones de conservación, utilizando una formulación en variables primarias sobre un dominio computacional en el cual se encuentran mallas desplazadas.

La estructura de algoritmo simple está compuesta de dos partes; la suposición de un campo de presiones que facilita la obtención de una distribución de velocidades y la corrección de estas distribuciones cumpliendo con la ecuación de continuidad de manera iterativa hasta llegar a una solución correcta [11].

Estos métodos de solución vienen preestablecidos en el programa de simulación de OpenFOAM, pero existen más opciones que pueden ser utilizadas dependiendo del caso, en el que se incluyen métodos numéricos de mayor orden de exactitud.

\section{2 Análisis experimental}

Para la realización del montaje experimental se tomaron las siguientes condiciones que deben cumplir los fluidos y materiales empleados:

La prueba se realiza hasta llegar al estado estacionario, es decir, el medio poroso seco se llenaba hasta que absorbiera la humedad. La prueba se iniciaba hasta que el valor del caudal fuera constante la velocidad se mantenía constante. 
Las pérdidas volumétricas fueron calculadas para estimar el caudal, una pequeña parte del flujo se fugaba en el dispositivo.

Se utilizó un recipiente de gran altura, el cual producía un flujo a través del medio poroso debido a la fuerza gravitacional, no se considera el flujo forzado por energía mecánica química, eléctrica, térmica o de otra naturaleza cualquiera.

El medio permeable debe estar saturado, sin presencia de aire para evitar la condición de multifluido o multifases, asegurando la valoración de la permeabilidad y el movimiento del fluido por los poros del medio permeable.

La estabilidad del agua en los piezómetros se toma como indicador necesario y suficiente para aceptar la condición de flujo laminar.

La relación lineal entre la velocidad de descarga y la pérdida de presión por unidad de longitud a través del material, se toma como indicativo de que el flujo a través del medio es laminar.

El medio permeable debe ser homogéneo e isotrópico, con el fin de permitir el análisis del flujo unidireccional.

Las características físicas y químicas de los medios deben permanecer constantes: el líquido no puede reaccionar con el medio, y la porosidad y la permeabilidad de este no deben cambiar durante el ensayo. Las reacciones químicas pueden dar lugar a cambios en la porosidad, ya sea por cementación o por disolución, y por lo tanto pueden cambiar la permeabilidad del medio junto con la aplicación de fuerzas externas, que dan lugar a cambios en la relación de vacíos [12].

En el caso de que otros efectos sean apreciables como el flujo gravitacional, se entiende el producido exclusivamente por efecto de la atracción gravitacional. Debido a que dependiendo el funcionamiento otros tipos de energía pueden ser considerados, ya sea mecánica, térmica, química, eléctrica o de tensión superficial. Se deben calcular los tipos de energía para cada caso. La regla técnica de Darcy, de aplicarse en estos casos por analogía, además debería estudiarse los coeficientes de permeabilidad específicos para cada energía de transporte o tipo de flujo. Así podría hablarse de permeabilidad osmótica, permeabilidad electro-osmótica, permeabilidad capilar, etc. [12].

\subsubsection{Montaje experimental}

El permeámetro es puesto a punto mediante los siguientes pasos:
1. El montaje se realiza tamizando una muestra de arena uniforme y se utiliza un tamiz para dejar el mismo tamaño de grano, Fig. 1.

2. Dos discos son utilizados para nivelar y ejercer presión al medio poroso dentro del permeámetro.

3. La estructura del permeámetro es ajustada mediante espárragos, también son instaladas válvulas de cierre y apertura a la entrada y salida del mismo, Fig. 2.

4. El agua se pone a circular en la arena seleccionada hasta que esta llegue al punto de saturación (los espacios vacíos son ocupados por agua), también las pérdidas volumétricas son cuantificadas. El agua fluye hasta llegar al estado estacionario en el que la continuidad del fluido se mantiene, es decir, la misma cantidad de agua que entra, sale, Fig. 3.

5. El flujo de agua se mantiene constante mediante un sistema de nivel, el agua fluye únicamente por efecto gravitacional y el tubo tiene un diámetro de un tamaño considerable en el que es posible despreciar los efectos de capilaridad, Fig. 4.

6. Finalmente, la cantidad de fluido es medida con un recipiente utilizado para este propósito, un cronómetro es utilizado para registrar el tiempo, y mediante una rejilla es medido el cambio de presión entre la entrada y la salida [13].

\subsubsection{Ley de Darcy}

La Ley de Darcy consiste en estudiar las propiedades de un flujo de agua a través de un lecho de arena, variando la longitud de la muestra y la presión del agua en los planos superficial e inferior. La Fig. 5 muestra la construcción de un permeámetro con altura constante, donde el caudal es $Q$ y está dada en $\mathrm{m}^{3} / \mathrm{s}$, el área de la sección transversal del filtro es $\mathrm{A}$ dado en $\mathrm{m}^{2}$, la velocidad del flujo está dada en $\mathrm{m} / \mathrm{s}$, la diferencia de niveles o cabeza es $\Delta$ h y está dada por el cambio de altura entre manómetros y la longitud del empaquetamiento es dado por $\Delta \mathrm{l}[9]$.

Darcy descubrió que el cambio de altura era proporcional a la longitud que tuviera el lecho poroso.

La velocidad del flujo se calcula con (8) y la conductividad hidráulica utilizando (9). 


$$
\begin{gathered}
u=\frac{q}{A} \\
u=K \frac{\Delta h}{\Delta L}
\end{gathered}
$$

Los siguientes supuestos se toman: la velocidad es constante en cualquier sección transversal, el medio a través del cual se produce el flujo es un medio poroso y la porosidad del medio está definida como la fracción de volumen vacío de aire sobre el volumen total. El valor de la porosidad varía en función de la temperatura y el contenido de agua almacenado. La porosidad se calcula mediante (10). La densidad de la masa concentrada de sólido se determina tomando la cantidad de masa seca y el volumen que ocupa la masa compactada, y la densidad a granel se calcula determinado la masa de una muestra del medio poroso en el fluido y se mide su volumen cuando este es afectado por la fuerza gravitacional [15].

$$
\varphi=1-\frac{\rho_{g r}}{\rho_{\text {sol }}}
$$

Fig. 1. TAMIZADO DEL MEDIO POROSO

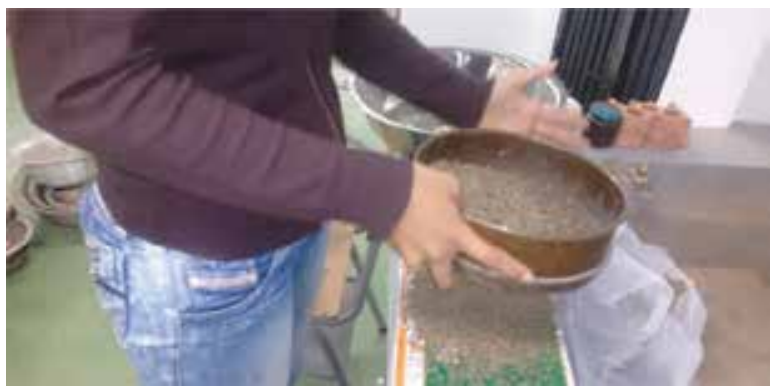

Fuente: Los autores.

Fig. 2. DISCOS DE COMPACTACIÓN

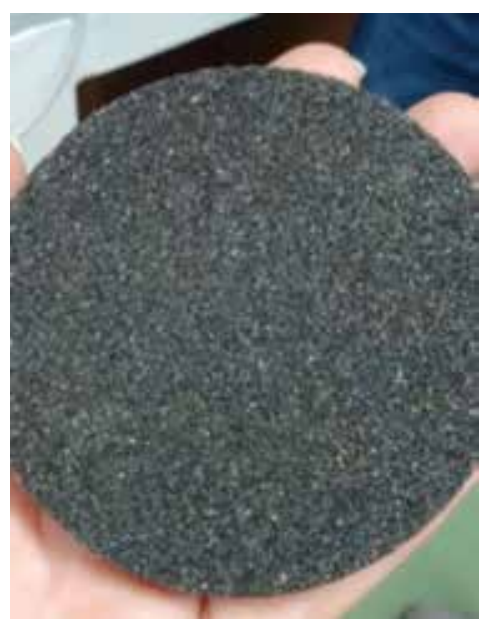

Fuente: Los autores.
Fig. 3. FLUJO EN ESTADO ESTACIONARIO

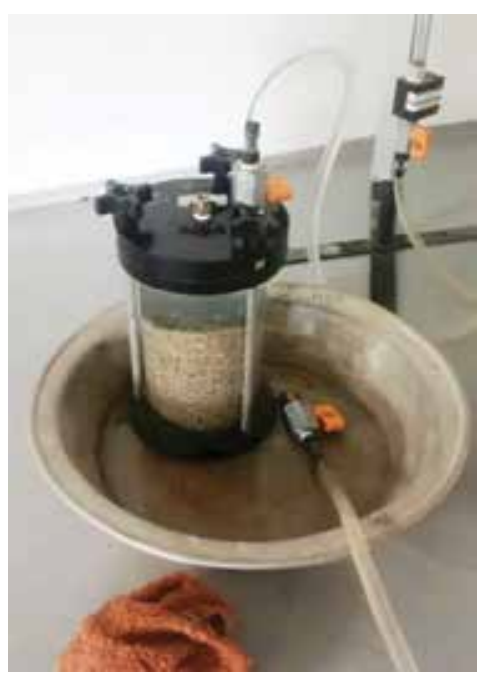

Fuente: Los autores.

Fig. 4. NIVEL DEL AGUA CONSTANTE

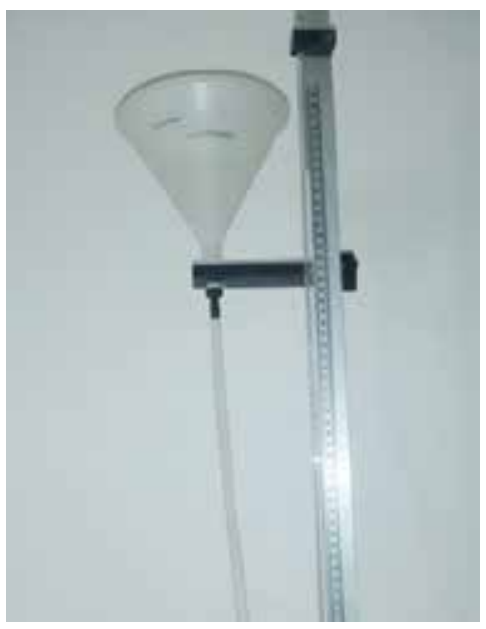

Fuente: Los autores.

Fig. 5. NIVEL DEL AGUA CONSTANTE

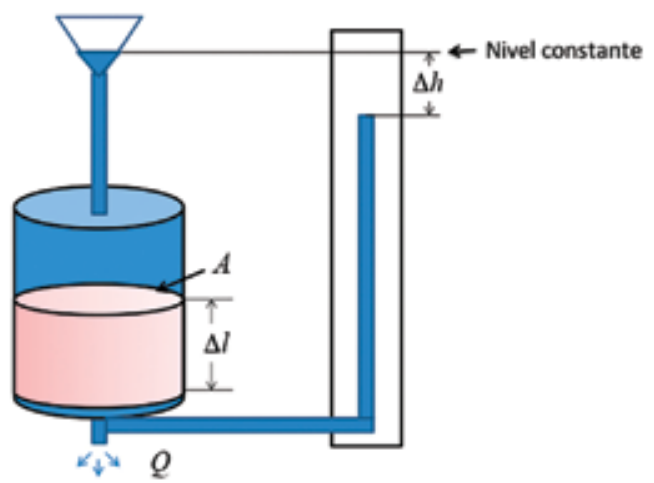

Fuente: Los autores. 
El flujo es en régimen laminar el cual se determina calculando el número de Reynolds. El número de Reynolds se calcula como la relación entre las fuerzas inerciales y las fuerzas viscosas según (11).

$$
\operatorname{Re}=\frac{D_{h} V_{s}}{\nu}
$$

Tomando la ecuación de Bernoulli para calcular el valor de como se muestra en (12). El valor de $\mathrm{h}$ es ajustado para que permanezca constante y el valor del cambio de altura se dice que se produce únicamente por los cambios de presión.

$$
h=\frac{P}{\gamma}+Z+\frac{V^{2} / 2}{2_{g}}
$$

Finalmente, el caudal se calcula con (13)

$$
Q=-\frac{K A}{\gamma} \frac{P_{1}-P_{2}}{L}
$$

El valor del peso específico sobre la constante de permeabilidad es proporcional a la viscosidad dinámica y la permeabilidad (propiedad de empaquetamiento) es función de la porosidad como se muestra en (14) y la viscosidad dinámica es función de la temperatura.

$$
\frac{K}{\gamma}=\frac{k(\varphi)}{\mu(T)}
$$

Remplazando (14) en (13) se logra (15) obteniendo la ley de Darcy, la cual se utilizó en el modelo numérico en términos de la velocidad mostrada en (15):

$$
Q=-\frac{k(\varphi) A}{\mu(T)} \frac{P_{1}-P_{2}}{L}
$$

\section{RESULTADOS}

Para las dos fases presentadas: la fase del modelo numérico y la fase 2 del análisis experimental, se muestra la descripción a continuación.

\subsection{Resultados del modelo numérico}

El modelo numérico se resuelve con las condiciones de contorno que se organizaron en los siguientes bloques: frente, atrás, paredes, paredes del medio poroso, entrada y salida. $\checkmark$ El bloque del frente, atrás, paredes y paredes del medio poroso tienen las siguientes condiciones: presión desconocida con una condición Zero gradient, lo cual indica que tiene un máximo o un mínimo; y para la velocidad se toma condición de no deslizamiento.

$\checkmark$ El bloque de entrada tenía las siguientes condiciones: presión desconocida con una condición de Zero gradient, lo cual indica un máximo o un mínimo; y flujo volumétrico con un valor de $0,1 \mathrm{~m}^{3} / \mathrm{s}$.

$\checkmark$ El bloque de salida tenía las siguientes condiciones: presión conocida, la cual se estima con los valores de la iteración anterior; y la velocidad es desconocida con una condición inletOutlet.

$\checkmark$ Con base en la temperatura, el medio poroso y el ducto donde este se encuentra tienen un aislamiento térmico, en el que la entrada y la salida la temperatura es de $20^{\circ} \mathrm{C}$.

En la Fig. 6, se muestra la escala de presión que arrojó el programa de simulación donde la presión se mantiene cercana a la presión del ambiente [16].

En la Fig. 7, se muestra la escala de velocidad donde el valor a la salida es cercano a cero, ya que el medio poroso en este caso se comporta como un tampón.

Como podemos observar en la Fig. 8, la presión disminuye de forma casi lineal, con una velocidad que presenta pequeñas variaciones en la dirección del flujo.

Los elementos se cambiaron desde 22 a 72 y las variaciones en la velocidad fueron inferiores al 1\%, como se muestra en la Fig. 9, también se variaron el número de elementos con respecto al radio y la velocidad se comparte de manera igual, esta no incrementa.

En la Fig. 10 y Fig. 11, de la presión cambiando la geometría del medio poroso, el campo de presión es proporcional al largo, y se obtuvo la siguiente ecuación con respecto al largo.

En la Fig. 12, podemos observar el perfil de velocidad y de la presión donde la velocidad tiene una diferencia del frente con respecto a las paredes de $54 \%$, esto se debe a la condición de no deslizamiento. 
Fig. 6. ESCALA DE PRESIÓN

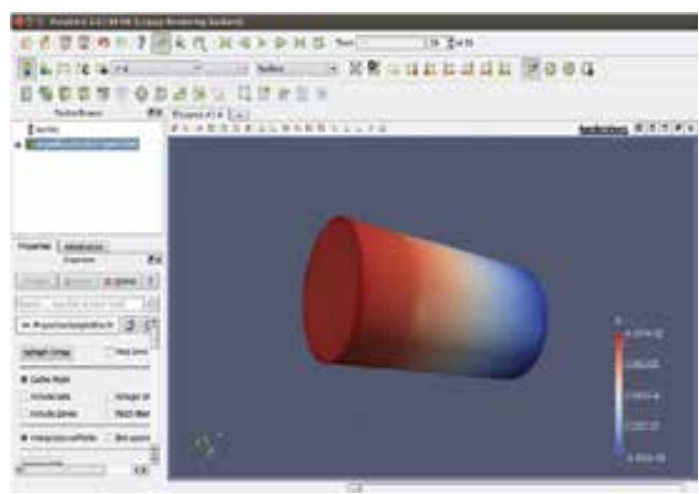

Fuente: Los autores.

Fig. 7. ESCALA DE VELOCIDAD

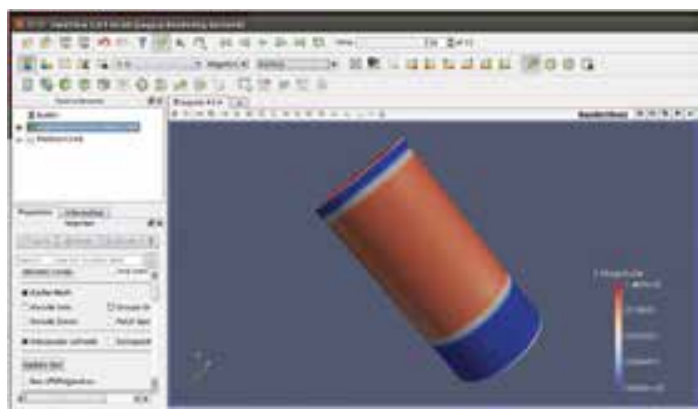

Fuente: Los autores.

Fig. 8. PRESIÓN Y VELOCIDAD EN EL PUNTO CENTRAL DEL PERMEÁMETRO

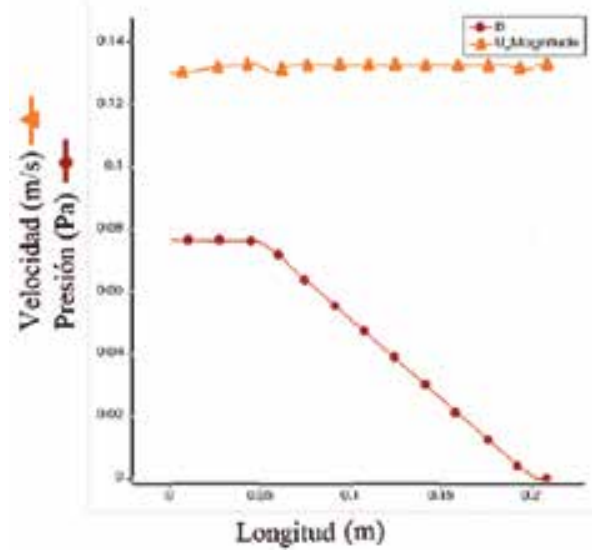

Fuente: Los autores.

Fig. 9. VARIACIÓN DE LA VELOCIDAD RESPECTO AL NÚMERO DE CELDAS

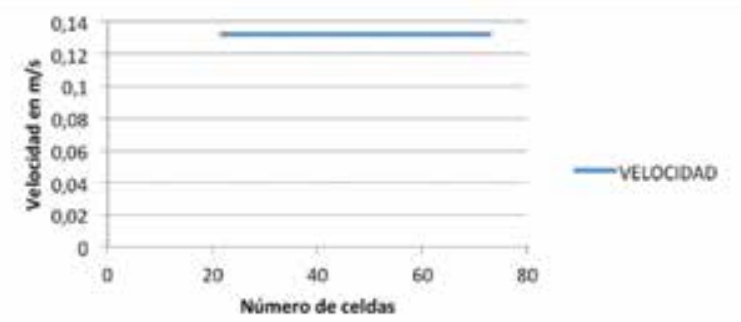

Fuente: Los autores.
Fig. 10. VARIACIÓN DE LA VELOCIDAD Y PRESIÓN EN EL CENTRO DEL DUCTO
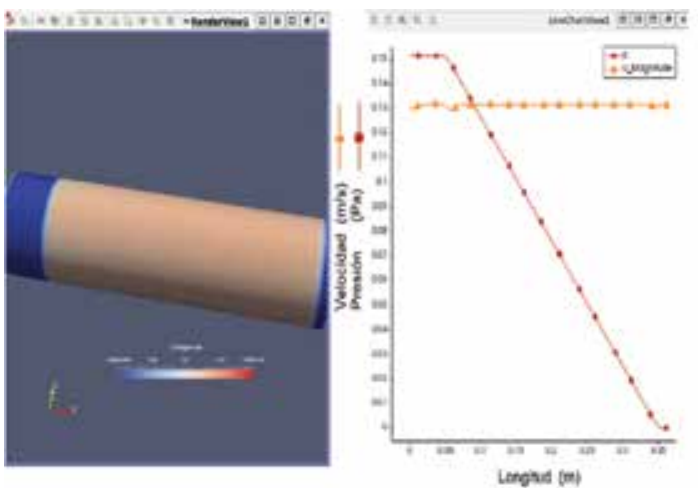

Fuente: Los autores.

Fig. 11. VARIACIÓN DE LA CAÍDA DE PRESIÓN RESPECTO AL LARGO

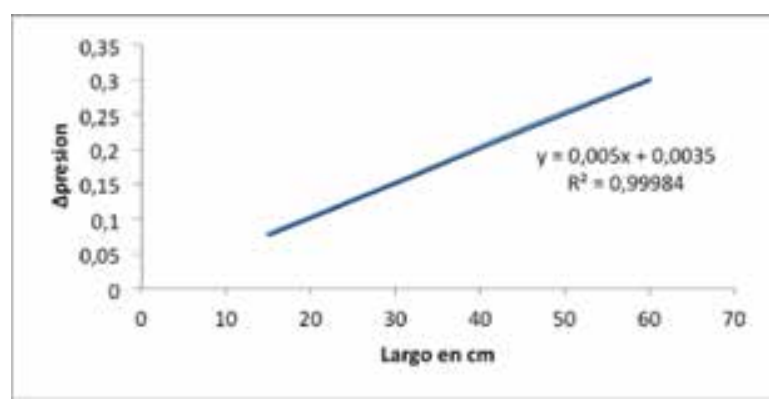

Fuente: Los autores.

Fig. 12. PERFIL DE VELOCIDAD Y PRESIÓN EN EL CENTRO DEL DUCTO

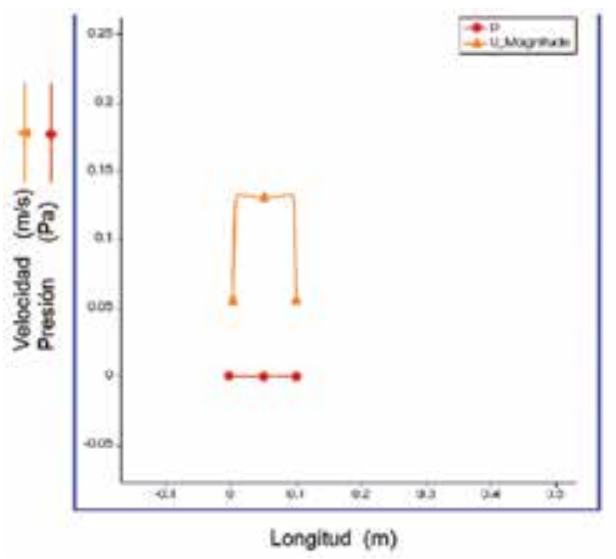

Fuente: Los autores.

\subsection{Resultados del análisis experimental}

La prueba se realiza con un permeámetro que funciona en las siguientes condiciones, con un diámetro de $74,71 \mathrm{~mm}$, una altura del medio poroso de $10 \mathrm{~cm}$ y el espesor del recipiente es de 6,84 mm. El número de Reynolds es de 280 según (16) que permite verificar que el régimen está en flujo laminar. 


$$
\mathrm{Re}=\frac{0,95 * 10^{-3} \mathrm{~m}^{3} * 4}{57,7 \mathrm{~s} \pi * 0,7471 \mathrm{~m}^{*} 1 * 10^{-6} \mathrm{~m}^{2} / \mathrm{s}}=280
$$

La conductividad hidráulica o coeficiente de permeabilidad (17) define la facilidad con la que el flujo de agua atraviesa el suelo, la cual se calcula acorde al procedimiento descrito en este documento. El valor del coeficiente de permeabilidad está en el rango de arenas muy finas, limos orgánicos e inorgánicos, mezclas de arena y depósitos de arcilla estratificada [17], [18], [9].

$$
\begin{gathered}
K=\frac{\forall L}{t \Delta h A} \quad(17) \\
K=\frac{0,95 * 10^{-3} \mathrm{~m}^{3} * 0,1 \mathrm{~m}^{* 4} 4}{57,7 \mathrm{~s}^{*} 0,962 \mathrm{~m} * \pi *(0,7471)^{2} \mathrm{~m}^{2}} \\
=3,91 x 10^{-4} \frac{\mathrm{cm}}{\mathrm{s}}
\end{gathered}
$$

Para el valor de conductividad hidráulica de $3,91 \times 10^{-4} \frac{\mathrm{cm}}{\mathrm{s}}$, el valor del número de Reynolds es de 280. En redes hidráulicas, sistemas de refrigeración, suelos arenosos, sistemas de purificación de agua, sistemas de generación de movimiento (motores Stirling) y filtros utilizados en aspiradoras, con números de Reynolds inferiores a 2000, estos sistemas pueden tener los siguientes factores de diseñado, variando 1) el caudal, 2) el diámetro y 3) el material poroso.

El caudal se varió para los valores del número de Reynolds de 30 a 1900. La Fig. 13, muestra que el gradiente de presión disminuye a medida que se incrementa el caudal. Esto es útil para conocer el comportamiento en los sistemas hidráulicos en los que se presentan variación en el caudal, por ejemplo un sistema con bombas en paralelo.

El diámetro varió de $30 \mathrm{~mm}$ a $80 \mathrm{~mm}$ como se muestra Fig. 14, y el cambio de presión se incrementó a mayor diámetro con concavidad negativa. Este factor es importante cuando se cambian las tuberías.

El cambio de presión es dependiente de la conductividad hidráulica, en la Fig. 15, se identifica que para un incremento en el porcentaje de densidad a granel varíe entre el 40 y el $80 \%$, este cambio es equivalente a utilizar distintos tipos de filtros, en este lecho poroso se observó la disminución del gradiente de presión acorde con la Ley de Darcy.

Se determinó la caída de presión en el permeámetro en el momento del montaje experimental.

La simulación en OpenFOAM arrojó los siguientes datos: viscosidad dinámica $\left(0.890 * 10^{-3} \mathrm{~N} . \mathrm{s} /\right.$ $\mathrm{m}^{2}$ ), coeficiente de Darcy $2.56 * 10^{5} \mathrm{~s} / \mathrm{m}$ (valor recíproco), número de celdas en OpenFOAM con las siguientes dimensiones (entrada del ducto: $5 \mathrm{~cm}$, lecho poroso: $15 \mathrm{~cm}$, salida: $1 \mathrm{~cm}$ y diámetro del medio poroso $7 \mathrm{~cm}$ ), siendo estos valores generalizados para este sistema.

Fig. 13. VARIACIÓN DE LA PRESIÓN EN FUNCIÓN DEL CAUDAL

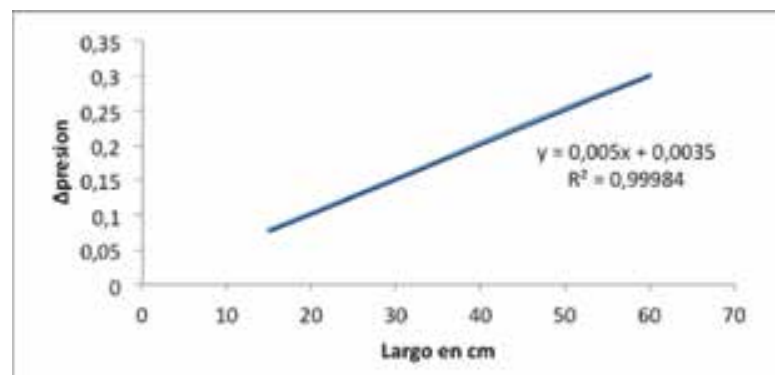

Fuente: Los autores.

Fig. 14. VARIACIÓN DE LA PRESIÓN EN FUNCIÓN DEL DIÁMETRO

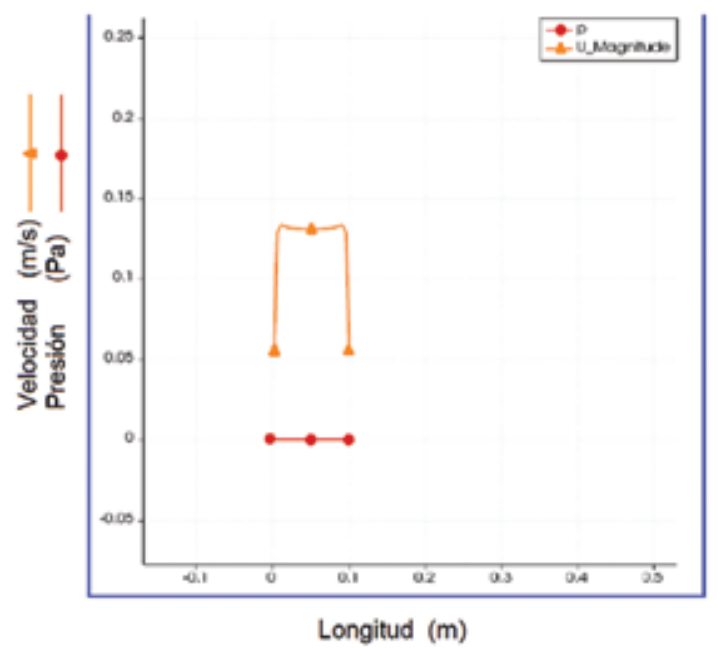

Fuente: Los autores.

Fig. 15. VARIACIÓN DE LA PRESIÓN EN FUNCIÓN DEL PORCENTAJE DE DENSIDAD A GRANEL

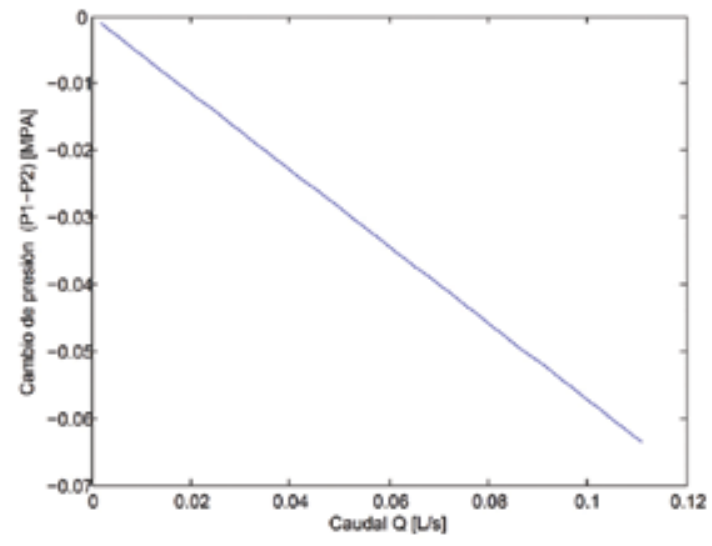

Fuente: Los autores. 


\section{CONCLUSIONES}

Del montaje experimental se obtuvo la conductividad hidráulica que es igual $3,91 \times 10^{-4} \mathrm{~cm} / \mathrm{s}$.

Se determinó que el medio poroso estaba en el rango de arenas muy finas y depósito de arcilla estratificada, lo cual fue fundamental para la implementación, utilización y simulación en el programa OpenFOAM.

De las simulaciones se encontró:

1. Que al disminuir el coeficiente de permeabilidad se necesita un mayor gradiente de presión para que el fluido pueda atravesar el ducto.

2. Cuando se varía la longitud del permeámetro se encontró que no se alcanza a desarrollar el perfil de velocidad.

3. Que la caída de presión en el ducto varía linealmente con el largo del lecho poroso, además cuando se cambia el número de celdas, las variaciones eran inferiores al $1 \%$, lo cual fue debido a bajas velocidades para un $\mathrm{Re}=$ 67,4 , se obtuvo un valor satisfactorio para las 880 celdas.

4. La ley de Darcy se estudió con cambios de caudal, área transversal del filtro y densidad a granel.

5. Cuando el caudal se incrementa, el gradiente de presión caía con pendiente negativa.

6. A mayor área transversal del filtro para un diámetro entre 30 y $80 \mathrm{~mm}$, el gradiente de presión se incrementara en forma cuadrática con concavidad negativa.

7. Cuando la densidad del granel se aumenta entre el 30 y $80 \%$, el valor de la presión decrece en forma curva con concavidad negativa.

\section{REFERENCIAS}

[1] L. de, D. Conductividad, and E. de Darcy, "Conductividad," Geol. Salamanca, 2014.

[2] M. Biot, "Theory of propagation of elastic waves in a fluid_saturated porous solid. I. Lowfrequ ency range," J. Acoust. Soc. Am., 1956.

[3] F. D. Salvador, "Validez de la ecuación binómica de Forchheimer en flujo convergente en un medio poroso Validity of Forchheimer binomial equation in convergent flow," RIHA, vol. 33, no. 1, pp. 18-29, 2012.
[4] J. Lizeth and P. Velandia, "Simulación numérica del flujo bifásico agua-petróleo," Revista Fuentes, vol. 11, No. 2, pp. 99-109, 2013.

[5] P. Leclaire and T. Cohen, "Observation of two longitudinal and two transverse waves in a frozen porous medium," J., 1995.

[6] J. Santos and D. Sheen, "Derivation of a Darcy's law for a porous medium composed of two solid phases saturated by a single-phase fluid: A homogenization approach," Transp. Porous Media, 2008.

[7] A. Ibáñez, M. Ramón, G. Blanquer, J. Manuel, "El uso del permeámetro de laboratorio." RiuNet.

[8] R. Juncosa and F. S. Calvete, "Modelos de flujo multifásico no isotermo y transporte reactivo multicomponente en medios porosos: 1. formulación físico matemática," Ing. del agua, 2002.

[9] D. Gabriels, D. Lobo, and M. Pullido, "Metodos para determinar la conductividad hidraulica saturada y no saturada de los suelos," Venesuelos, no. 14, pp. 7-22, 2006.

[10] Greenshields, Christopher J. Openfoam user guide, OpenFOAM Foundation Ltd, version 5.0, pp. 73-134, 2017.

[11] J. Xamán, M. Gijón-Rivera, Dinámica de fluidos computacional para ingenieros. Amazon.es Libros, 2016.

[12] J. F. Romaña García, "Los límites de la Ley de Darcy," bdigital.unal.edu.co, p. 114, 2014.

[13] M. Durán Pinzón, J. E. Páez Arango, and P. J. García Guarín, "Flow through soils in a homogeneous porous media," in 3rd International Congress of Mechanical Engineering and Agricultural Science, p. 8, 2017.

[14] I. R. Tupak Obando, Hidrogeología Ley de Darcy y ecuaciones fundamentales flujo en medios porosos flujo en medios porosos, 2010.

[15] P. J. García Guarín, Caracterización del comportamiento térmico de un secador híbrido solar-biomasa de túnel tipo Hohenheim con base en experimentación y simulación computacional, Universidad Nacional de Colombia, 2016.

[16] M. Durán Pinzón, J. E. Páez Arango, and P. J. García Guarín, "Modelado numérico para flujos en un medio poroso homogéneo," XI Congr. Colomb. Métodos Numéricos, p. 8, 2017.

[17] S. Angelone, M. Garibay, and M. Casaux, Permeabilidad de suelos, 2006.

[18] Y. Bárcena and J. Hurtado, Ensayos de permeabilidad en materiales de baja de permeabilidad compactados, 2011. 